\title{
iGb3: to be or not to be?
}

DOI:

10.1038/nri2082
The authors of two reports published in the Proceedings of the National Academy of Sciences of the USA suggest that, contrary to popular belief, the glycosphingolipid iGb3 (isoglobotrihexosylceramide) is not the ligand involved in the positive selection of invariant natural killer $\mathrm{T}(i \mathrm{NKT})$ cells in the thymus.

$\mathrm{CD} 1$ is a non-polymorphic MHC-class-I-like transmembrane

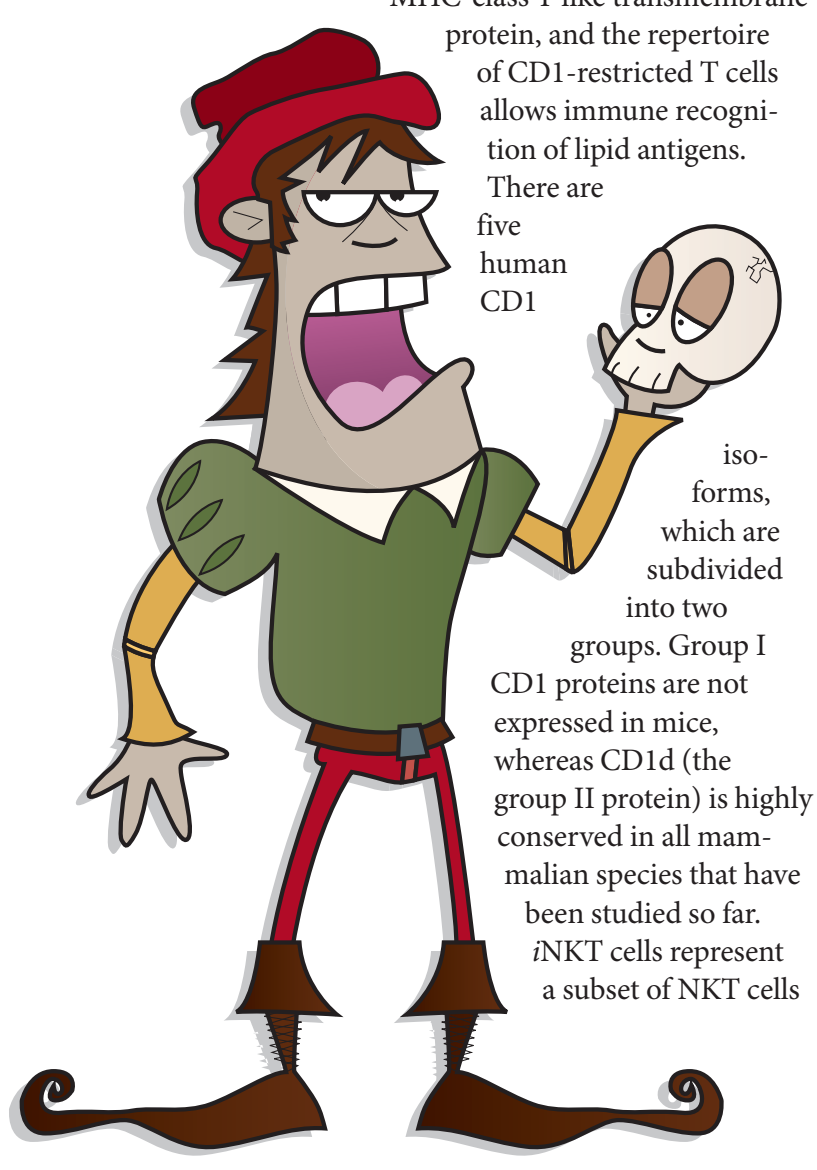

that express an invariant T-cell receptor (TCR) $\alpha$-chain and a restricted set of $\beta$-chains, and are selected in the thymus by the presentation of an endogenous lipid bound to CD1d on $\mathrm{CD} 4^{+} \mathrm{CD} 8^{+}$cortical thymocytes. It is thought that this selecting lipid ligand is a glycosphingolipid - more recent evidence points to iGb3, which has also been implicated in the peripheral activation of $i$ NKT cells by dendritic cells (DCs).

But doubts have been raised about the role of iGb3 in the positive selection of $i \mathrm{NKT}$ cells in the thymus in vivo. Decreased numbers of $i \mathrm{NKT}$ cells have been observed in some mouse models of glycosphingolipid storage diseases that do not primarily interfere with the formation of iGb3. Moreover, the presence of iGb3 in the thymus would be required if it were to be involved in $i$ NKT-cell selection, yet there has been no biochemical data showing that isoglobo-series glycosphingolipids are expressed in mouse or human thymi.

To address this issue, Speak et al. used a highly sensitive high-pressure liquid chromatography (HPLC) assay to study the distribution of iGb3 in mouse and human tissues. iGb3 was detected in mouse dorsal root ganglion, but not in any other mouse or human tissue analysed, including the thymus or DCs. In an accompanying report, Porubsky et al. generated mice deficient in iGb3 synthase to directly assess the role of iGb3 as a selecting ligand for $i \mathrm{NKT}$ cells in mice. igb3s $\mathrm{s}^{-/-}$mice showed normal $i$ NKT-cell development, function, numbers and usage of the TCR V $\beta$ chain. The authors could not rule out the possibility that other glycosphingolipids could take on the role of iGb3 in its absence, although they think it is unlikely as TCR V $\beta$ chain usage and $i$ NKT-cell development were normal, and there were no compensatory changes in other glycosphingolipids in these mice.

Speak et al. note that their findings do not question that iGb3 can serve as a ligand for $i$ NKT cells in vitro but, together, the results from these two reports do not support a role for iGb3 in the positive selection of $i$ NKT cells in the mouse or human thymus in vivo.

So, is iGb3 to be or not to be the selecting ligand for $i$ NKT cells in the thymus? Although there is no definitive answer yet, these papers show that iGb3 is not the only selecting $i$ NKT-cell ligand.

Sharon Ahmad

ORIGINAL RESEARCH PAPERS Speak, A. O. et al. Implications for invariant natural killer T cell ligands due to the restricted presence of isoglobo trihexosylceramide in mammals. Proc. Natl Acad. Sci. USA 19 March 2007 (doi:10.1073/ pnas.0607285104)| Porubsky, S. et al. Normal development and function of invariant natural killer T cells in mice with isoglobotrihexosylceram ide (iGb3) deficiency. Proc. Natl Acad. Sci. USA 19 March 2007 (doi: 10.1073/pnas.0611139104) 